\title{
Shifting finite time stability and boundedness design for continuous-time LPV systems
}

\author{
Damiano Rotondo, Fatiha Nejjari, Vicenç Puig
}

\begin{abstract}
In this paper, the problem of designing a parameter-scheduled state-feedback controller is investigated. In particular, the concepts of finite time stability (FTS) and finite time boundedness (FTB) are extended, introducing their shifting counterparts. By introducing new scheduling parameters, the controller can be designed in such a way that different values of these parameters imply different characteristics of the finite time stability/boundedness property. In this way, the performance of the control system can be varied during its operation. The problem is analyzed in the continuous-time LPV case, even though the developed theory could be also applied to LTI systems. The design conditions are feasibility problems involving linear matrix inequalities (LMIs) that can be solved efficiently using available solvers. Results obtained in simulation demonstrate the effectiveness and the relevant features of the proposed approach.
\end{abstract}

\section{INTRODUCTION}

The idea of finite time stability (FTS), as introduced by [1], allows to analyze or design control systems that present some constraints on the state response without being necessarily stable [2]. In fact, when the behavior of the system over a fixed time interval is of interest, a system could be referred to as stable when, given some initial conditions, the state remains within some desired bounds in such time interval, and unstable when it does not [3]. It is worth recalling that the idea of FTS is independent from the classical Lyapunov asymptotic stability, since a system can be finite time stable but not asymptotically stable, and vice versa [4].

Another concept which is strongly related to FTS is finite time boundedness (FTB), which takes into account norm bounded disturbances affecting the system [3]. In particular, a system is said to be FTB if, given a bound on the initial condition and a characterization of the set of admissible disturbances, the state remains within the prescribed bounds for all the disturbances in the set. FTB implies FTS, but the converse is not true.

The last decade has seen a lot of development of these two concepts. For example, the output feedback design case has been considered in [5]. In such case, the design is divided in two steps: the synthesis of a state feedback controller, and

This work has been funded by the Spanish Ministry of Science and Technology through the projects CICYT SHERECS (ref. DPI2011-26243) and CICYT ECOCIS (ref. DPI2013-48243-C2-1-R), by AGAUR through the contract FI-DGR 2014 (ref. 2014FI_B1 00172) and by the DGR of Generalitat de Catalunya (SAC group Ref. 2014/SGR/374).

D. Rotondo, F. Nejjari and V. Puig are with Automatic Control Department, Universitat Politècnica de Catalunya (UPC), Rambla de Sant Nebridi, 11, 08222 Terrassa, Spain. e-mail: \{damiano.rotondo, fatiha.nejjari, vicenc.puig\}@upc.edu the design of a state observer capable of retaining the finitetime properties guaranteed by the controller. The authors of [6]-[8] have extended these definitions to different type of systems (e.g. singular ones) with impulsive effect. The definition of finite-time $\mathcal{H}_{\infty}$ control has been presented in [9] for dealing with systems subject to time-varying normbounded exogenous disturbance. Finally, other works have dealt with finite-time quantized guaranteed cost fuzzy control [10] and FTS of delay systems [11].

In the last decades, gain-scheduling control techniques have consolidated as an efficient answer to analysis and synthesis problems for nonlinear systems [12]. The strength of these techniques consists in the fact that the properties of the nonlinear systems are checked on the basis of a collection of linear systems, that is also used for designing the controller. This is done in a divide and conquer fashion so that well established linear methods can be applied to nonlinear problems. Among the approaches that have proved to be successful for performing gain-scheduling control, there is the linear parameter varying (LPV) paradigm, introduced by Shamma [13]. This class of systems is important because gain-scheduling control of nonlinear systems can be performed according to the LPV paradigm by embedding the nonlinearities in the varying parameters that depend on some endogenous signals, e.g. some system states [14] (in this case, the system is named quasi-LPV). The LPV approach has been successfully applied in many situations [15], [16], among which finite time stability [17].

Recently, the idea of shifting specifications has been introduced as an extension of some classical control problems [18], [19]. This idea relies on introducing some varying parameters, or using the existing ones, to schedule the controller such that different values of these parameters imply different performances. From a practical point of view, reasons for which this problem can be of interest include all situations where some performance degradation could be desirable, e.g. high/low-gain control, control of systems with saturation nonlinearities [20], graceful performance degradation for active fault-tolerant control [21] and actuator health degradation avoidance [22].

The goal of this paper is to further explore the possibilities offered by shifting specifications, introducing the concepts of shifting finite time stability and shifting finite time boundedness. These concepts are developed theoretically, and a numerical example is used to illustrate the relevant features that distinguish them from other results available in the literature.

This paper is structured as follows. Section II recalls some 
definitions and known results that will be used throughout the paper. The main theoretical results of this work, i.e. the design using shifting finite time boundedness and shifting finite time stability specifications, are presented in Section III. An example of application of the proposed approach is shown in Section IV. Finally, Section V outlines the main conclusions.

Notation: If a matrix $M \in \mathbb{R}^{n \times n}$ is symmetric, then $M \in$ $\mathbb{S}^{n \times n}$. A matrix $M \in \mathbb{S}^{n \times n}$ is said positive definite $(M \succ$ $0)$ if all its eigenvalues are positive, and negative definite $(M \prec 0)$ if all its eigenvalues are negative. For a generic vector $v$, its length is denoted by $n_{v}$, i.e. $v \in \mathbb{R}^{n_{v}} . I$ and $O$ denote the identity matrix and the zero matrix of appropriate dimensions, respectively.

\section{Preliminaries}

The idea of finite time stability concerns the boundedness of the state of a system over a finite time interval for given initial conditions. This concept has been formalized in [3], extending the original formulation provided by [1], as follows.

Definition 1: The continuous-time linear system

$$
\dot{x}(t)=A(t) x(t)
$$

is said to be finite time stable (FTS) with respect to $\left(c_{1}, c_{2}, T, R\right)$, with $c_{2}>c_{1}>0$ and $R \succ O$ if

$$
x(0)^{T} R x(0) \leq c_{1} \Rightarrow x(t)^{T} R x(t)<c_{2} \quad \forall t \in[0, T]
$$

On the other hand, the idea of state boundedness is more general and concerns the behavior of the state in presence of external disturbances [3].

Definition 2: The continuous-time linear system

$$
\dot{x}(t)=A(t) x(t)+G(t) w(t)
$$

is said to be finite time bounded (FTB) with respect to $\left(c_{1}, c_{2}, T, R, d\right)$, with $c_{2}>c_{1}>0, R \succ O$ and $d>0$ if

$$
\left\{\begin{array}{l}
x(0)^{T} R x(0) \leq c_{1} \\
w(t)^{T} w(t) \leq d
\end{array} \quad \Rightarrow x(t)^{T} R x(t)<c_{2} \quad \forall t \in[0, T]\right.
$$

Notice that FTS can be recovered as a special case of FTB when $w=0$.

Let us now recall the definition of linear parameter varying (LPV) systems. Following the terminology of [23], LPV systems are linear time varying (LTV) plants whose statespace matrices are fixed functions of some vector of varying parameters $\theta(t) \in \Theta \subset \mathbb{R}^{n_{\theta}}$. By replacing (1) and (3) with

$$
\begin{gathered}
\dot{x}(t)=A(\theta(t)) x(t) \\
\dot{x}(t)=A(\theta(t)) x(t)+G(\theta(t)) w(t)
\end{gathered}
$$

and by requiring that (2) and (4) hold $\forall \theta \in \Theta$, the characterization of FTS and FTB for LPV systems is obtained.

An important class of LPV systems are the polytopic ones, for which the state-space matrices satisfy the following property

$$
\left(\begin{array}{c}
A(\theta(t)) \\
G(\theta(t))
\end{array}\right)=\sum_{i=1}^{N} \alpha_{i}(\theta(t))\left(\begin{array}{c}
A_{i} \\
G_{i}
\end{array}\right) \quad \forall \theta \in \Theta
$$

with

$$
\sum_{i=1}^{N} \alpha_{i}(\theta(t))=1 \quad \alpha_{i}(\theta(t)) \geq 0
$$

The following lemma provides a sufficient condition for the FTB of a continuous-time LPV system.

Lemma 1: The continuous-time LPV system (6) is FTB with respect to $\left(c_{1}, c_{2}, T, R, d\right)$ if, letting $\tilde{Q}_{1}=R^{-1 / 2} Q_{1} R^{-1 / 2}$, there exist positive scalars $\alpha, \lambda_{1}, \lambda_{2}, \lambda_{3}$ and two positive definite matrices $Q_{1} \in \mathbb{S}^{n_{x} \times n_{x}}$ and $Q_{2} \in \mathbb{S}^{n_{w} \times n_{w}}$ such that the following conditions hold $\forall \theta \in \Theta$

$$
\begin{gathered}
\left(\begin{array}{cc}
A(\theta) \tilde{Q}_{1}+\tilde{Q}_{1} A(\theta)^{T}-\alpha \tilde{Q}_{1} & G(\theta) Q_{2} \\
Q_{2} G(\theta)^{T} & -\alpha Q_{2}
\end{array}\right) \prec O \\
\lambda_{1} I \prec Q_{1} \prec I \\
\lambda_{2} I \prec Q_{2} \prec \lambda_{3} I \\
\left(\begin{array}{ccc}
c_{2} e^{-\alpha T} & \sqrt{c_{1}} & \sqrt{d} \\
\sqrt{c_{1}} & \lambda_{1} & 0 \\
\sqrt{d} & 0 & \lambda_{2}
\end{array}\right) \succ O
\end{gathered}
$$

Proof: The proof is based on showing that, once defined:

$$
V(x(t), w(t))=x(t)^{T} \tilde{Q}_{1}^{-1} x(t)+w(t)^{T} Q_{2}^{-1} w(t)
$$

the following holds $\forall \boldsymbol{\theta} \in \Theta$ :

$$
\dot{V}(x(t), w(t))<\alpha V(x(t), w(t))
$$

The details are omitted due to space limitations, but follow the reasoning provided by [3].

Conditions similar to those of Lemma 1 can be obtained for the case of FTS for continuous-time LPV systems.

Corollary 1: The continuous-time LPV system (5) is FTS with respect to $\left(c_{1}, c_{2}, T, R\right)$ if, letting $\tilde{Q}_{1}=R^{-1 / 2} Q_{1} R^{-1 / 2}$, there exist positive scalars $\alpha, \lambda_{1}$ and a positive definite matrix $Q_{1} \in \mathbb{S}^{n_{x} \times n_{x}}$ such that the following conditions hold $\forall \theta \in \Theta$

$$
\begin{gathered}
A(\theta) \tilde{Q}_{1}+\tilde{Q}_{1} A(\theta)^{T}-\alpha \tilde{Q}_{1} \prec O \\
\lambda_{1} I \prec Q_{1} \prec I \\
\left(\begin{array}{cc}
c_{2} e^{-\alpha T} & \sqrt{c_{1}} \\
\sqrt{c_{1}} & \lambda_{1}
\end{array}\right) \succ O
\end{gathered}
$$

Proof: It is a direct consequence of Lemma 1, when $G(\theta(t))=O$ and $d=0$.

\section{MAin Results}

In this paper, we consider the problem of designing a continuous-time LPV state-feedback control law

$$
u(t)=K(\theta(t), p(t)) x(t)
$$

where $p(t)$ is a vector of scheduling parameters defined in the following, so as to satisfy one of the following specifications

- shifting finite time boundedness

- shifting finite time stability

Remark 1: Despite in this paper the problem of control design using shifting specification is considered for the case of LPV systems, the proposed method is useful for LTI systems too. In this case, the controller is scheduled by 
means of the vector of parameters $p(t)$, such that, even though the plant to be controlled is LTI, the overall system is LPV and the mathematical reasoning developed hereafter can be applied. The reason to do so is that in this way the performance of the closed-loop system can be varied in time according to some criterium, e.g. energetic issues.

\section{A. Shifting finite time boundedness}

Given the following LPV system

$$
\dot{x}(t)=A(\theta(t)) x(t)+B u(t)+G(\theta(t)) w(t)
$$

it is wished to design the controller (18) such that the resulting closed-loop system is shifting finite time bounded (SFTB) with respect to $\left(c_{1}(p(t)), c_{2}(p(t)), T(p(t)), R, d(p(t))\right) \forall p \in \Pi$, where the definition of SFTB for continuous-time systems is given hereafter.

Definition 3: The continuous-time LPV system (6) is said to be shifting finite time bounded (SFTB) with respect to $\left(c_{1}(p(t)), c_{2}(p(t)), T(p(t)), R, d(p(t))\right)$, with $c_{2}(p(t))>$ $c_{1}(p(t))>0, d(p(t))>0 \forall p \in \Pi$ and $R \succ O$ if

$\left\{\begin{array}{l}x\left(t_{0}\right)^{T} R x\left(t_{0}\right) \leq c_{1}\left(p_{0}\right) \\ w(t)^{T} w(t) \leq d\left(p_{0}\right) \\ p(t)=p_{0} \quad \forall t \in\left[t_{0}, t_{0}+T\left(p_{0}\right)\right]\end{array} \Rightarrow \begin{array}{l}x(t)^{T} R x(t)<c_{2}\left(p_{0}\right) \\ \forall t \in\left[t_{0}, t_{0}+T\left(p_{0}\right)\right]\end{array}\right.$

Then, the following theorem provides conditions for the system (19) controlled by (18) to be SFTB.

Theorem 1: The continuous-time LPV system (19) under the control law (18) is SFTB with respect to $\left(c_{1}(p(t)), c_{2}(p(t)), T(p(t)), R, d(p(t))\right)$ if, letting $\tilde{Q}_{1}=$ $R^{-1 / 2} Q_{1} R^{-1 / 2}$, there exist a positive scalar $\alpha$, positive functions $\lambda_{1}(p), \lambda_{2}(p), \lambda_{3}(p)$ and positive definite matrices $Q_{1} \in$ $\mathbb{S}^{n_{x} \times n_{x}}$ and $Q_{2} \in \mathbb{S}^{n_{w} \times n_{w}}$ such that the following conditions hold $\forall \theta \in \Theta$ and $\forall p \in \Pi$

$$
\begin{gathered}
\left(\begin{array}{cc}
A_{c l}(\theta, p) \tilde{Q}_{1}+\tilde{Q}_{1} A_{c l}(\theta, p)^{T}-\alpha \tilde{Q}_{1} & G(\theta) Q_{2} \\
Q_{2} G(\theta)^{T} & -\alpha Q_{2}
\end{array}\right) \prec O \\
\lambda_{1}(p) I \prec Q_{1} \prec I \\
\lambda_{2}(p) I \prec Q_{2} \prec \lambda_{3}(p) I \\
\left(\begin{array}{ccc}
c_{2}(p) e^{-\alpha T(p)} & \sqrt{c_{1}(p)} & \sqrt{d(p)} \\
\sqrt{c_{1}(p)} & \lambda_{1}(p) & 0 \\
\sqrt{d(p)} & 0 & \lambda_{2}(p)
\end{array}\right) \succ O \\
A_{c l}(\theta, p)=A(\theta)+B K(\theta, p)
\end{gathered}
$$

Proof: From Definition 3, by introducing the new time variable $\tilde{t}=t-t_{0}$, (20) becomes

$$
\left\{\begin{array}{l}
x(0)^{T} R x(0) \leq c_{1}\left(p_{0}\right) \\
w(0)^{T} w(0) \leq d\left(p_{0}\right) \\
p(\tilde{t})=p_{0} \quad \forall \tilde{t} \in\left[0, T\left(p_{0}\right)\right]
\end{array} \quad \Rightarrow \quad \begin{array}{cc}
x(\tilde{t})^{T} R x(\tilde{t})<c_{2}\left(p_{0}\right) \\
\forall \tilde{t} \in\left[0, T\left(p_{0}\right)\right]
\end{array}\right.
$$

Since $p(\tilde{t})$ is constant during the considered time interval, it follows that in order to obtain (26), the property of finite time boundedness, as defined in Definition 1, must hold $\forall p \in \Pi$. By replacing (18) into (19), the LPV form (6) is recovered, with a state matrix given by $A(\theta)+B K(\theta, p)$. Hence, by considering that Lemma 1 should hold $\forall p \in \Pi$, (21)-(24) are obtained, completing the proof.

From a practical point of view, Theorem 1 is useless because it relies on the satisfaction of infinite constraints. This difficulty can be alleviated by fixing a value for $\alpha$ under the assumption that the matrices $A(\theta)$ and $G(\theta)$ are polytopic as in (7)-(8), and that

$$
\left(\begin{array}{c}
\sqrt{c_{1}(p)} \\
c_{2}(p) e^{-\alpha T(p)} \\
\sqrt{d(p)}
\end{array}\right)=\sum_{j=1}^{P} \pi_{j}(p)\left(\begin{array}{c}
\kappa_{1 j} \\
\kappa_{2 j} \\
\kappa_{3 j}
\end{array}\right) \quad \forall p \in \Pi
$$

with

$$
\sum_{j=1}^{P} \pi_{j}(p)=1 \quad \pi_{j}(p) \geq 0
$$

Then, it is possible to reduce (21)-(24) to a finite number of Linear Matrix Inequalities (LMIs), as stated in the following corollary.

Corollary 2: Let $\tilde{Q}_{1}=R^{-1 / 2} Q_{1} R^{-1 / 2}$, fix $\alpha>0$, and find positive scalars $\lambda_{1 j}, \lambda_{2 j}, \lambda_{3 j}, j=1, \ldots, P$, positive definite matrices $Q_{1} \in \mathbb{S}^{n_{x} \times n_{x}}$ and $Q_{2} \in \mathbb{S}^{n_{w} \times n_{w}}$, and $P \cdot N$ matrices $\Gamma_{i j} \in \mathbb{R}^{n_{u} \times n_{x}}$ such that the following conditions hold $\forall i=$ $1, \ldots, N$ and $\forall j=1, \ldots, P$

$$
\begin{gathered}
\left(\begin{array}{rr}
A_{i} \tilde{Q}_{1}+B \Gamma_{i j}+\tilde{Q}_{1} A_{i}^{T}+\Gamma_{i j}^{T} B^{T}-\alpha \tilde{Q}_{1} & G_{i} Q_{2} \\
Q_{2} G_{i}^{T} & -\alpha Q_{2}
\end{array}\right) \prec O \\
\lambda_{1 j} I \prec Q_{1} \prec I \\
\lambda_{2 j} I \prec Q_{2} \prec \lambda_{3 j} I \\
\left(\begin{array}{ccc}
\kappa_{2 j} & \kappa_{1 j} & \kappa_{3 j} \\
\kappa_{1 j} & \lambda_{1 j} & 0 \\
\kappa_{3 j} & 0 & \lambda_{2 j}
\end{array}\right) \succ O
\end{gathered}
$$

Then, the LPV system (19) under control law (18) with

$$
K(\theta(t), p(t))=\sum_{i=1}^{N} \alpha_{i}(\theta(t)) \sum_{j=1}^{P} \pi_{j}(p(t)) K_{i j}
$$

and

$$
K_{i j}=\Gamma_{i j} \tilde{Q}_{1}^{-1}
$$

is SFTB with respect to:

$$
\left(c_{1}(p(t)), c_{2}(p(t)), T(p(t)), R, d(p(t))\right)
$$

Proof: Due to a basic property of matrices [24], any linear combination of (29) with non-negative coefficients is negative definite. Similarly, any linear combination of (32) with non-negative coefficients is positive definite. For the same reason, the inequalities (30)-(31) would still hold for any linear combination with non-negative coefficients. Hence, using the coefficients provided by (8) and (28), and taking into account (7), (27), (33), $\Gamma_{i j}=K_{i j} \tilde{Q}_{1}$ and

$$
\left(\begin{array}{l}
\lambda_{1}(p) \\
\lambda_{2}(p)
\end{array}\right)=\sum_{j=1}^{P} \pi_{j}(p)\left(\begin{array}{c}
\lambda_{1 j} \\
\lambda_{2 j}
\end{array}\right)
$$

(21)-(24) are obtained. 
Remark 2: In some cases, the input matrix $B$ in (19) is parameter-varying. In these cases, a prefiltering of the input $u(t)$ would lead to defining a new system with a constant input matrix $\tilde{B}$, as proposed in [25]. More specifically, defining a new input $\tilde{u}(t)$ such that

$$
\begin{gathered}
\dot{x}_{u}(t)=A_{u} x_{u}(t)+B_{u} \tilde{u}(t) \\
u(t)=C_{u} x_{u}(t)
\end{gathered}
$$

where $A_{u}$ is stable, the resulting system would be

$$
\begin{aligned}
\left(\begin{array}{c}
\dot{x}(t) \\
\dot{x}_{u}(t)
\end{array}\right) & =\left(\begin{array}{cc}
A(\theta(t)) & B(\theta(t)) C_{u} \\
O & A_{u}
\end{array}\right)\left(\begin{array}{c}
x(t) \\
x_{u}(t)
\end{array}\right) \\
& +\left(\begin{array}{c}
O \\
B_{u}
\end{array}\right) \tilde{u}(t)+\left(\begin{array}{c}
G(\theta(t)) \\
O
\end{array}\right) w(t)
\end{aligned}
$$

such that the new input matrix is parameter-independent.

\section{B. Shifting finite time stability}

Given the following LPV system

$$
\dot{x}(t)=A(\theta(t)) x(t)+B u(t)
$$

the objective is to design the controller (18) such that the resulting closed-loop system is shifting finite time stable (SFTS) with respect to $\left(c_{1}(p(t)), c_{2}(p(t)), T(p(t)), R\right) \forall p \in$ $\Pi$, where the definition of SFTS for continuous-time systems is given hereafter.

Definition 4: The continuous-time LPV system (5) is said to be shifting finite time stable (SFTS) with respect to $\left(c_{1}(p(t)), c_{2}(p(t)), T(p(t)), R\right)$ with $c_{2}(p(t))>c_{1}(p(t))>0 \forall p \in \Pi$ and $R \succ O$ if

$\left\{\begin{array}{l}x\left(t_{0}\right)^{T} R x\left(t_{0}\right) \leq c_{1}\left(p_{0}\right) \\ p(t)=p_{0} \quad \forall t \in\left[t_{0}, t_{0}+T\left(p_{0}\right)\right]\end{array} \quad \Rightarrow \quad \begin{array}{l}x(t)^{T} R x(t)<c_{2}\left(p_{0}\right) \\ \forall t \in\left[t_{0}, t_{0}+T\left(p_{0}\right)\right]\end{array}\right.$

Then, the following theorem provides conditions for the system (39) controlled by (18) to be SFTS.

Theorem 2: The continuous-time LPV system (39) under the control law (18) is SFTS with respect to $\left(c_{1}(p(t)), c_{2}(p(t)), T(p(t)), R\right)$ if, letting $\tilde{Q}_{1}=R^{-1 / 2} Q_{1} R^{-1 / 2}$, there exist a positive scalar $\alpha$, a positive function $\lambda_{1}(p)$ and a positive definite matrix $Q_{1} \in \mathbb{S}^{n_{x} \times n_{x}}$ such that the following conditions hold $\forall \boldsymbol{\theta} \in \Theta$ and $\forall p \in \Pi$

$$
\begin{gathered}
A_{c l}(\theta, p) \tilde{Q}_{1}+\tilde{Q}_{1} A_{c l}(\theta, p)^{T}-\alpha \tilde{Q}_{1} \prec O \\
\lambda_{1}(p) I \prec Q_{1} \prec I \\
\left(\begin{array}{cc}
c_{2}(p) e^{-\alpha T(p)} & \sqrt{c_{1}(p)} \\
\sqrt{c_{1}(p)} & \lambda_{1}(p)
\end{array}\right) \succ O
\end{gathered}
$$

with $A_{c l}(\theta, p)$ defined as in (25).

Proof: The proof is similar to the one of Theorem 1, and thus is omitted.

As in the shifting finite time boundedness case, in order to reduce (41)-(43) to a finite number of constraints, $\alpha$ is fixed, and the assumptions that $A(\theta)$ is polytopic as in (7)-(8), and that

$$
\left(\begin{array}{c}
\sqrt{c_{1}(p)} \\
c_{2}(p) e^{-\alpha T(p)}
\end{array}\right)=\sum_{j=1}^{P} \pi_{j}(p)\left(\begin{array}{c}
\kappa_{1 j} \\
\kappa_{2 j}
\end{array}\right) \quad \forall p \in \Pi
$$

with coefficients $\pi_{j}(p)$ satisfying (28), are made, leading to the following corollary.

Corollary 3: Let $\tilde{Q}_{1}=R^{-1 / 2} Q_{1} R^{-1 / 2}$, fix $\alpha>0$, and find positive scalars $\lambda_{1 j}, j=1, \ldots, P$, and a positive definite matrix $Q_{1} \in \mathbb{S}^{n_{x} \times n_{x}}$ and $P \cdot N$ matrices $\Gamma_{i j} \in \mathbb{R}^{n_{u} \times n_{x}}$ such that the following conditions hold $\forall i=1, \ldots, N$ and $\forall j=1, \ldots, P$

$$
\begin{gathered}
A_{i} \tilde{Q}_{1}+B \Gamma_{i j}+\tilde{Q}_{1} A_{i}^{T}+\Gamma_{i j}^{T} B^{T}-\alpha \tilde{Q}_{1} \prec O \\
\lambda_{1 j} I \prec Q_{1} \prec I \\
\left(\begin{array}{cc}
\kappa_{2 j} & \kappa_{1 j} \\
\kappa_{1 j} & \lambda_{1 j}
\end{array}\right) \succ O
\end{gathered}
$$

Then, the LPV system (39) under the control law (18) with the matrix $K(\theta(t), p(t))$ calculated as in (33)-(34) is SFTS with respect to $\left(c_{1}(p(t)), c_{2}(p(t)), T(p(t)), R\right)$.

Proof: The proof is similar to the one of Corollary 2, and thus is omitted.

Remark 3: Also in this case, if the input matrix $B$ in (39) is parameter-varying, a prefiltering of the input $u(t)$ using (36)-(37) would lead to an augmented system with parameter-independent input matrix.

\section{EXAMPLE}

The example used to illustrate the method proposed in this paper is a slight modification of the numerical example provided by [3]. Consider the LPV system (39) with matrices defined as follows

$$
\begin{gathered}
A(\theta(t))=\left(\begin{array}{cc}
0 & 1 \\
-2+\theta(t) & -1+\theta(t)
\end{array}\right) \\
B=\left(\begin{array}{ll}
0 & 1
\end{array}\right)^{T}
\end{gathered}
$$

where $\theta(t) \in[-10,0]$, and consider the problem of designing the controller (18) such that the resulting closed-loop system is SFTS with respect to $\left(1, c_{2}(p(t)), T(p(t)), I\right)$ with

$$
\begin{gathered}
c_{2}(p(t))=1.7+0.3(1-p(t)) \\
T(p(t))=0.5-0.1(1-p(t))
\end{gathered}
$$

First of all, notice that $A(\theta(t))$ can be described in the polytopic form

$$
A(\theta(t))=\alpha_{1}(\theta(t)) A_{1}+\alpha_{2}(\theta(t)) A_{2}
$$

with

$$
\begin{aligned}
& A_{1}=\left(\begin{array}{cc}
0 & 1 \\
-12 & -11
\end{array}\right) \quad A_{2}=\left(\begin{array}{cc}
0 & 1 \\
-2 & -1
\end{array}\right) \\
& \alpha_{1}(\theta(t))=-\frac{\theta(t)}{10} \quad \alpha_{2}(\theta(t))=1+\frac{\theta(t)}{10}
\end{aligned}
$$

Then, once a value $\alpha=1$ has been chosen, one can verify that

$$
c_{2}(p(t)) e^{-\alpha T(p(t))}=\pi_{1}(p(t)) \kappa_{21}+\pi_{2}(p(t)) \kappa_{22}
$$

with

$$
\begin{gathered}
\kappa_{21}=2 e^{-0.4} \kappa_{22}=1.7 e^{-0.5} \\
\pi_{1}(p(t))=\frac{c_{2}(p(t)) e^{-\alpha T(p(t))}-1.7 e^{-0.5}}{2 e^{-0.4}-1.7 e^{-0.5}}
\end{gathered}
$$




$$
\pi_{2}(p(t))=\frac{2 e^{-0.4}-c_{2}(p(t)) e^{-\alpha T(p(t))}}{2 e^{-0.4}-1.7 e^{-0.5}}
$$

The application of Corollary 3 leads to searching for positive scalars $\lambda_{11}$ and $\lambda_{12}, Q_{1} \succ O$ and matrices $\Gamma_{11}, \Gamma_{12}$, $\Gamma_{21}, \Gamma_{22}$ such that

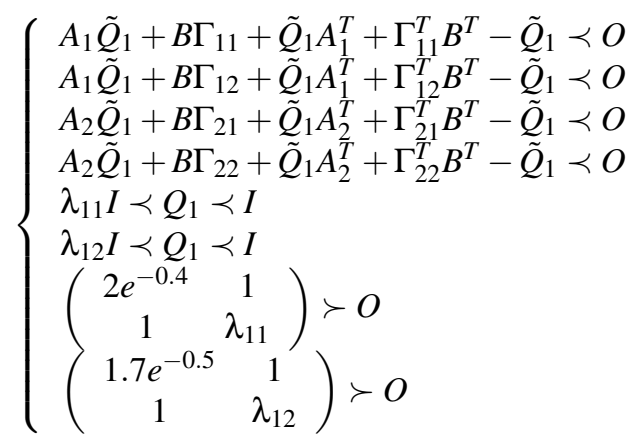

Using the YALMIP toolbox [26] with SeDuMi solver [27], the following solution has been found

$$
\begin{gathered}
K_{11}=K_{12}=\left(\begin{array}{ll}
10.9998 & 11.1948
\end{array}\right) \\
K_{21}=K_{22}=\left(\begin{array}{ll}
0.9998 & 1.1948
\end{array}\right)
\end{gathered}
$$

Simulations with $x(0)=\left(\begin{array}{cc}1 & 0\end{array}\right)^{T}$ and $x(0)=\left(\begin{array}{ll}0 & 1\end{array}\right)^{T}$ have shown that the obtained closed-loop systems satisfy the desired shifting specifications, as shown in Fig. 1 for different values of the parameter $p(t)$. However, notice that the LMI solver favors a conservative solution, where the gains corresponding to different values of the parameter $p(t)$ are the same. Hence, the closed-loop dynamics does not vary for different values of $p(t)$, as shown in Fig. 1 .

In order to obtain a less conservative solution, let us add the following LMIs to (59)

$$
\left\{\begin{array}{l}
2 s_{1}^{\min } \tilde{Q}_{1}-A_{1} \tilde{Q}_{1}-B \Gamma_{11}-\tilde{Q}_{1} A_{1}^{T}-\Gamma_{11}^{T} B^{T} \prec O \\
2 s_{1}^{\min } \tilde{Q}_{1}-A_{2} \tilde{Q}_{1}-B \Gamma_{21}-\tilde{Q}_{1} A_{2}^{T}-\Gamma_{21}^{T} B^{T} \prec O \\
2 s_{2}^{\min } \tilde{Q}_{1}-A_{1} \tilde{Q}_{1}-B \Gamma_{12}-\tilde{Q}_{1} A_{1}^{T}-\Gamma_{12}^{T} B^{T} \prec O \\
2 s_{2}^{\min } \tilde{Q}_{1}-A_{2} \tilde{Q}_{1}-B \Gamma_{22}-\tilde{Q}_{1} A_{2}^{T}-\Gamma_{22}^{T} B^{T} \prec O \\
A_{1} \tilde{Q}_{1}+B \Gamma_{11}+\tilde{Q}_{1} A_{1}^{T}+\Gamma_{11}^{T} B^{T}-2 s_{1}^{\max } \tilde{Q}_{1} \prec O \\
A_{2} \tilde{Q}_{1}+B \Gamma_{21}+\tilde{Q}_{1} A_{2}^{T}+\Gamma_{21}^{T} B^{T}-2 s_{1}^{\max } \tilde{Q}_{1} \prec O \\
A_{1} \tilde{Q}_{1}+B \Gamma_{12}+\tilde{Q}_{1} A_{1}^{T}+\Gamma_{12}^{T} B^{T}-2 s_{2}^{\max } \tilde{Q}_{1} \prec O \\
A_{2} \tilde{Q}_{1}+B \Gamma_{22}+\tilde{Q}_{1} A_{2}^{T}+\Gamma_{22}^{T} B^{T}-2 s_{2}^{\max } \tilde{Q}_{1} \prec O
\end{array}\right.
$$

that correspond to a shifting pole placement [18] in the LMI region

$$
\mathcal{D}=\left\{z \in \mathbb{C}: s_{\min }(p(t))<\operatorname{Re}(z)<s_{\max }(p(t))\right\}
$$

with

$$
\begin{aligned}
& s_{\min }(p(t))=s_{2}^{\min }+\left(s_{1}^{\min }-s_{2}^{\min }\right)(1-p(t)) \\
& s_{\max }(p(t))=s_{2}^{\max }+\left(s_{1}^{\max }-s_{2}^{\max }\right)(1-p(t))
\end{aligned}
$$

with $s_{1}^{\min }=0.5, s_{2}^{\min }=-100, s_{1}^{\max }=100, s_{2}^{\max }=0$ (notice that this specification corresponds to asking the closed-loop system to be unstable for $p(t)=0$, and stable for $p(t)=1$ ).

Then, a solution to the LMIs (59) and (62) is

$$
K_{11}=\left(\begin{array}{ll}
9.4305 & 11.6247
\end{array}\right)
$$
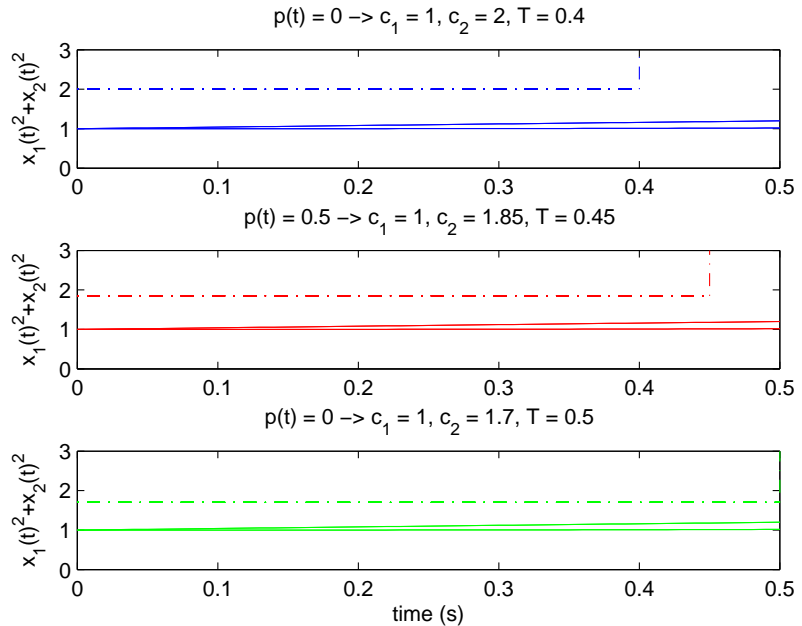

Fig. 1. $x_{1}(t)^{2}+x_{2}(t)^{2}$ using the proposed shifting finite time stability approach.

$$
\begin{aligned}
K_{12} & =\left(\begin{array}{ll}
-0.5695 & 1.6247
\end{array}\right) \\
K_{21} & =\left(\begin{array}{ll}
22.9370 & -83.1982
\end{array}\right) \\
K_{22} & =\left(\begin{array}{ll}
12.9370 & -93.1982
\end{array}\right)
\end{aligned}
$$

The relevant feature of this solution is that the characteristics of the closed-loop system would vary according to the value of $p(t)$, always satisfying the SFTS specification, as shown in Fig. 2. Also, if we take a deeper look at the results obtained for $p(t)=0$ (see Fig. 3), it can be seen that the closed-loop system with $p(t)=0$ satisfies the SFTS with respect to $(1,2,0.4, I)$, corresponding to $p(t))=0$. However, this is not the case with respect to $(1,1.85,0.45, I)$ and $(1,1.7,0.5, I)$, that correspond to $p(t)=0.5$ and $p(t)=1$, respectively.

This feature is in accordance with the developed theory and distinguishes the proposed method from other results available in the literature.

\section{CONCLUSIONS}

In this paper, the problem of designing a parameterscheduled state-feedback controller that satisfies a new kind of specifications, referred to as shifting finite time boundedness and shifting finite time stability, has been investigated. The problem has been analyzed in the continuous-time LPV case, even though the developed theory could also be applied to LTI systems. The design conditions are feasibility problems involving LMIs that can be solved efficiently using available solvers. In contrast with the classical concepts of finite time boundedness and finite time stability, the design using shifting specifications allows to select different characteristics for different values of the scheduling parameter $p$, thus allowing to vary online the control system performance. Results obtained in simulation with an LPV numerical example have demonstrated the effectiveness and the relevant features of the proposed approach. 

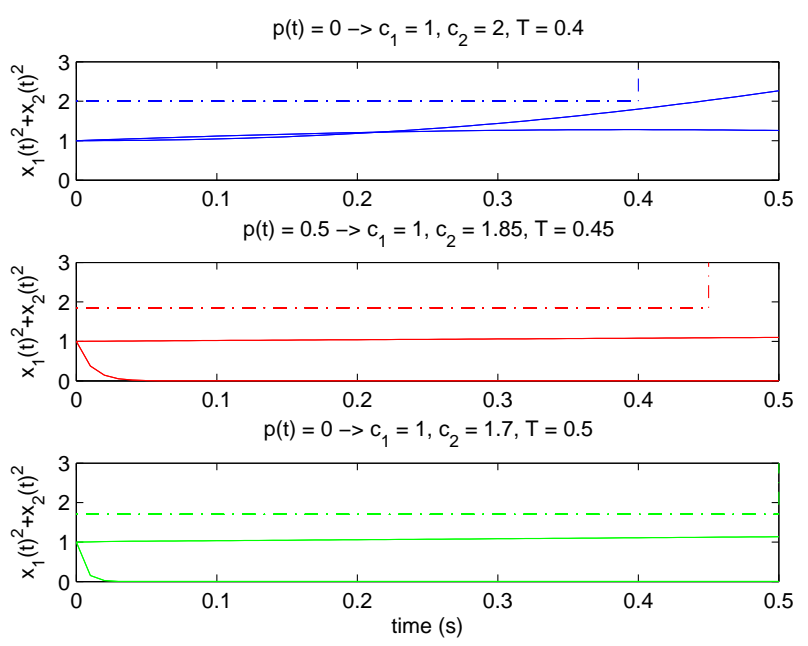

Fig. 2. $x_{1}(t)^{2}+x_{2}(t)^{2}$ using the proposed shifting finite time stability approach adding shifting pole placement constraints.

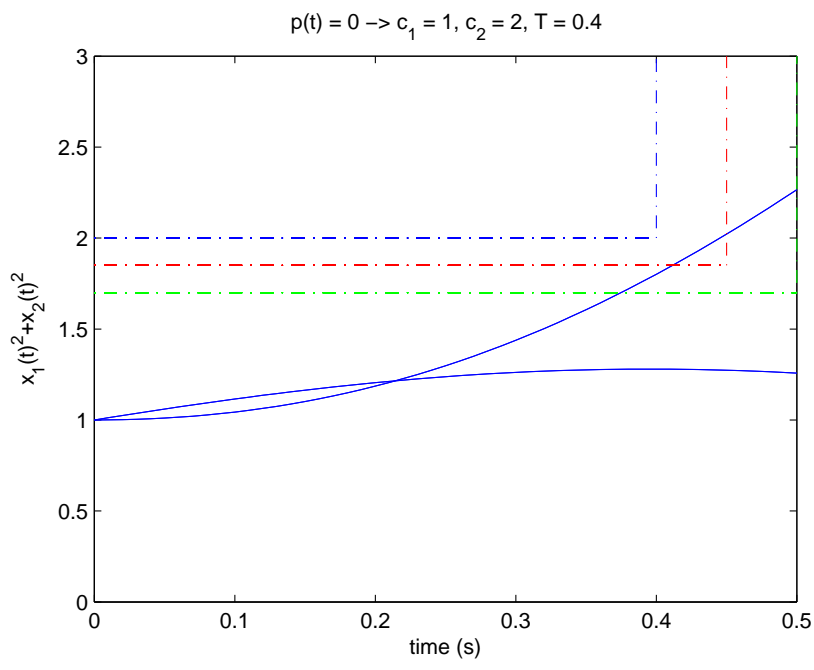

Fig. 3. $x_{1}(t)^{2}+x_{2}(t)^{2}$ using the proposed shifting finite time stability approach adding shifting pole placement constraints $(p(t)=0)$.

\section{REFERENCES}

[1] P. Dorato, "Short time stability in linear time-varying systems," in Proceedings of the IRE International Convention Record, Part 4, 1961, pp. 83-87.

[2] P. Dorato, C. T. Abdallah, and D. Famularo, "Robust finite-time stability design via linear matrix inequalities," in Proceedings of the 36th IEEE Conference on Decision and Control, San Diego, CA, USA, 1997, pp. 1305-1306.

[3] F. Amato, M. Ariola, and P. Dorato, "Finite-time control of linear systems subject to parametric uncertainties and disturbances," Automatica, vol. 37, pp. 1459-1463, 2001.

[4] F. Amato, M. Ariola, M. Carbone, and C. Cosentino, "Finite-time control of linear systems: a survey," in Current Trends in Nonlinear Systems and Control, L. Menini, L. Zaccarian, and C. T. Abdallah, Eds. Springer, 2006, pp. 195-213.

[5] F. Amato, M. Ariola, and C. Cosentino, "Finite-time stabilization via dynamic output feedback," Automatica, vol. 42, pp. 337-342, 2006.

[6] J. Feng, Z. Wu, and J. Sun, "Finite-time control of linear singular systems with parametric uncertainties and disturbances," Acta Automatica Sinica, vol. 31, no. 4, pp. 634-637, 2005.
[7] L. Liu and J. Sun, "Finite-time stabilization of linear systems via impulsive control," International Journal of Control, vol. 81, no. 6 , pp. 905-909, 2008.

[8] S. Zhao, J. Sun, and L. Liu, "Finite-time stability of linear timevarying singular systems with impulsive effects," International Journal of Control, vol. 81, no. 11, pp. 1824-1829, 2008.

[9] Q. Meng and Y. Shen, "Finite-time $\mathcal{H}_{\infty}$ control for linear continuous system with norm-bounded disturbance," Communications in Nonlinear Science and Numerical Simulation, vol. 14, pp. 1043-1049, 2009.

[10] D. Yang and K.-Y. Cai, "Finite-time quantized guaranteed cost fuzzy control for continuous-time nonlinear systems," Expert systems with applications, vol. 14, pp. 1043-1049, 2009.

[11] D. L. Debeljkovic, S. B. Stojanovic, and A. M. Jovanovic, "Finite-time stability of continuous time delay systems: Lyapunov-like approach with Jensen's and Coppel's Inequality," Acta Polytechnica Hungarica, vol. 10, no. 7, pp. 135-150, 2013.

[12] D. J. Leith and W. E. Leithead, "Survey of gain-scheduling analysis design," International Journal of Control, vol. 73, pp. 1001-1025, 2000.

[13] J. S. Shamma, "Analysis and Design of Gain Scheduled Control Systems," Ph.D. dissertation, Massachussets Institute of Technology, Departmenet of Mechanical Engineering, 1988.

[14] — "An overview of LPV systems," in Control of Linear Parameter Varying Systems with Applications, J. Mohammadpour and C. Scherer, Eds. Springer, 2012.

[15] C. Hoffmann and H. Werner, "A survey of linear parameter-varying control applications validated by experiments or high-fidelity simulations," IEEE Transactions on Control Systems Technology, vol. 23, no. 2, pp. 416-433, 2015.

[16] D. Rotondo, F. Nejjari, and V. Puig, "Quasi-LPV modeling, identification and control of a twin rotor MIMO system," Control Engineering Practice, vol. 21, pp. 829-846, 2013.

[17] Y. Shen, "Finite-time control of linear parameter-varying systems with norm-bounded exogenous disturbance," Journal of Control Theory and Applications, vol. 6, no. 2, pp. 184-188, 2008.

[18] D. Rotondo, F. Nejjari, and V. Puig, "A shifting pole placement approach for the design of parameter-scheduled state-feedback controllers," in Proceedings of the European Control Conference (ECC), 2013, pp. 1829-1834.

[19] — "Design of parameter-scheduled state-feedback controllers using shifting specifications," Journal of the Franklin Institute, vol. 352, no. 1, pp. 93-116, 2015.

[20] F. Wu, K. M. Grigoriadis, and A. Packard, "Anti-windup controller design using linear parameter-varying control methods," International Journal Of Control, vol. 73, pp. 1104-1114, 2000.

[21] Y. Zhang, J. Jiang, and D. Theilliol, "Incorporating performance degradation in fault tolerant control system design with multiple actuator failures," International Journal of Control, Automation, and Systems, vol. 6, no. 3, pp. 327-338, 2008.

[22] A. Khelassi, D. Theilliol, P. Weber, and J.-C. Ponsart, "Fault-tolerant control design with respect to actuator health degradation: An LMI approach," in Proceedings of the International Conference on Control Applications (CCA), 2011.

[23] J. Shamma and J. Cloutier, "A linear parameter-varying approach to gain scheduled missile autopilot design," in Proceedings of American Control Conference, 1992, pp. 1317-1321.

[24] R. A. Horn and C. R. Johnson, Matrix Analysis. Cambridge University Press, 1990

[25] P. Apkarian, P. Gahinet, and G. Becker, "Self-scheduled $H_{\infty}$ Control of Linear Parameter-Varying Systems: A Design Example," Automatica, vol. 31, no. 9, pp. 1251 - 1261, 1995.

[26] J. Löfberg, "YALMIP : a toolbox for modeling and optimization in MATLAB," in Proceedings of the CACSD Conference, Taipei, Taiwan, 2004

[27] J. F. Sturm, "Using SeDuMi 1.02, a MATLAB toolbox for optimization over symmetric cones," Optimization Methods and Software, vol. 1112 , pp. 625-653, 1999 . 